\title{
Femtocell-to-Femtocell Handoff Management in Dense Femtocellular Networks
}

\author{
Faisal A. Al-Shahin \\ State Audit Bureau, Kuwait. \\ * Corresponding author. Tel.:+965-99353353; email: alshahain@gmail.com \\ Manuscript submitted July 9, 2014; accepted April 11, 2015. \\ doi: 10.17706/ijcce.2015.4.5.346-353
}

\begin{abstract}
Femtocells are small consumer-installed base stations that enhance indoor cellular services coverage. Deploying a large number of such femtocells in a small area results in "Dense Femtocellular Networks." These networks face a number of problems, including handoff. Different scholars have put forward various prescriptions for resolving the handoff problem. In this paper, we propose a model to enhance and minimize the neighbor femtocell list for femtocell-to-femtocell handoff in a dense femtocellular network by adapting a user movement prediction technique based on femtocell location.
\end{abstract}

Key words: Dense femtocellular networks, femtocell, handoff, mobile station received signal strength.

\section{Introduction}

It is well-known that most data traffic and voice traffic originate from indoor mobile users [1]. However, due to the attenuation of the signal by walls and other factors, operators usually fail to provide high-quality service to home users, and many subscribers experience problems with poor indoor coverage. This problem highlights the need for a technology such as femtocells. A femtocell refers to an indoor, low-power, low-cost wireless access point with a short coverage area that connects mobile devices to a mobile operator's network using an existing broadband Internet connection to provide better indoor coverage and capacity [2]. A typical deployment of a femtocell network is shown in Fig. 1. As shown in Fig. 2, there are three main network elements common to any femtocell network architecture: (i) a home node B (HNB), also known as a femtocell access point (FAP), (ii) a security gateway (SeGW), and (iii) an HNB management system (HMS).

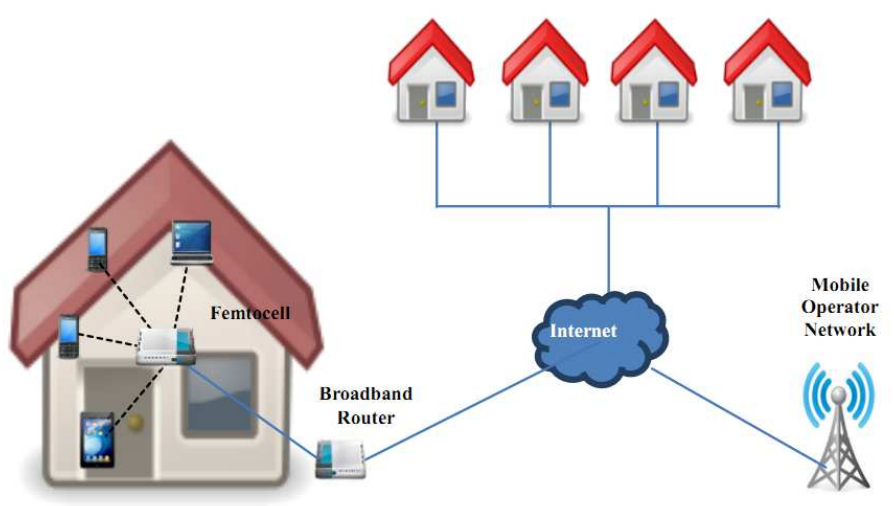

Fig. 1. Overview of a femtocell network. 


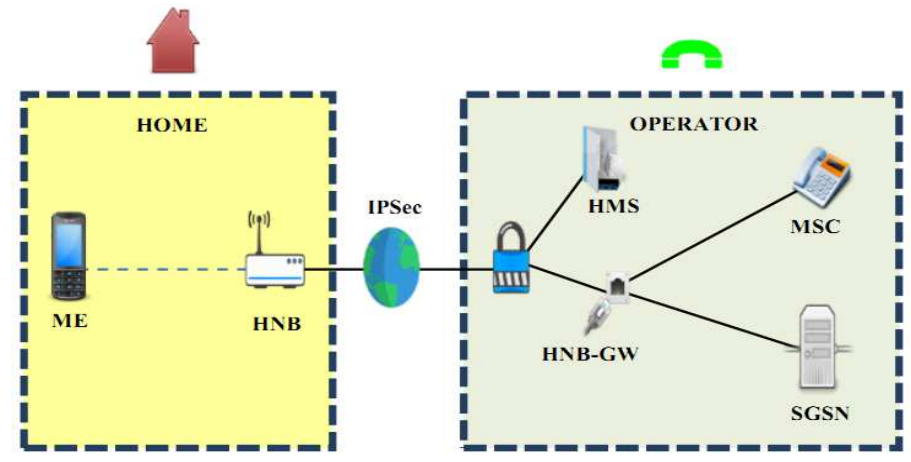

Fig. 2. Femtocell network architecture.

The HNB is the primary node in a femtocell network. It is located indoors at the user's location and is typically owned by the end user. It connects the mobile equipment to the operator's network over a secure tunnel through the Internet. The SeGW provides a secure link between the HNB and the mobile operator's core network, using standard Internet security protocols to authenticate and authorize femtocells and encrypt the traffic. The third main element in the femtocell network architecture, the HMS, is used for provisioning the HNB configuration data remotely using standards that are usually used for DSL modem configuration. It plays a significant role in activation and operational management by ensuring low-cost deployment and easy setup for users, with no on-site assistance required from the mobile operator [3].

Femtocell technology offers many benefits to both the user and the mobile network operator. In relation to the user, it can provide better indoor coverage, higher data performance, an improved multimedia experience, and higher voice quality. The benefits for the femtocell operator include increased network capacity, reduced capital costs, expanded revenue opportunities, and decreased backhaul costs. However, femtocell networks face a number of unique challenges. For example, interference can occur either between a macrocell and a femtocell or between femtocells. Access control is also an issue; restricted access is applied to allow only authorized users to access the femtocells. A particularly serious concern for femtocell deployment is handover/handoff. Handoff occurs when a user moves onto or off a femtocell. During handoff, seamless coverage and call continuity must be preserved. Handoff occurs either from macrocell to femtocell, from femtocell to macrocell, or from femtocell to femtocell.

In this paper, we focus on femtocell-to-femtocell handoff in dense femtocellular networks, in which a large number of femtocells are installed in a small area. The main goal of our technique is to minimize the neighbor femtocell list to facilitate the handoff between femtocells.

The rest of this paper is organized as follows. In Section 2, some works related to femtocell handoff are discussed. In Section 3 and Section 4, we analyze and criticize our motivational paper. The proposed model is presented in Section 5. Finally, we present our conclusions and suggestions for future work in Section 6 and Section 7, respectively.

\section{Related Works}

Different authors have proposed many solutions with regard to the issue of handoff in femtocells. In an empirical study, Shih-Jung Wu [4] suggested a way in which handoff could be managed between macrocells and femtocells in hybrid access mode. For femtocell-to-macrocell handoff, he compared the mobile velocity to two preset velocity levels and accordingly either checked the availability of bandwidth or used the received signal strength to decide whether there would be a handoff or not. For macrocell-to-femtocell handoff, he considered a hybrid access mode in which a mobile station is either a closed subscriber group or not. Accordingly, the received signal strength and velocity will be checked to decide whether the handoff will be executed given the bandwidth availability. 
On the other hand, Tiantian Bai et al. [5] focused on ways in which the handoff from macrocell to femtocell could be effectively managed based on decisions made by an entity named the HNB policy function (HNB PF). This entity uses measurement parameters, such as the received signal strength (RSS) and interference-to-noise ratio included in the handoff requests. It then interacts with the femtocells to obtain specific information and thus make the best decision about the target femtocell for the handoff. Once a handoff request is sent to the HNB PF using an RSS that is stronger than the serving macrocell, the HNB PF compares the information in the HNBs with the allowed closed subscriber group list from the mobile equipment. Results for different kinds of femtocells are given different priorities by the HNB PF. Accordingly, the HNB PF selects the optimal one, and the handoff is assigned to the most available femtocell.

Another algorithm, proposed by Chowdhury et al. [6], generates a femtocell list that contains a minimum number of neighbor femtocells to establish the femtocell-to-femtocell handoff in a dense femtocell network. The proposed scheme first considers the RSS level, and the frequency for each FAP is then allocated. Any femtocell that uses the same frequency as the serving FAP is eliminated. Finally, using the location information coordinated by the neighbor FAP, the hidden femtocells in the neighbor femtocell list are added.

As a different approach, a mobility pattern prediction algorithm was presented in [7] to avoid unnecessary macrocell-to-femtocell handoffs when the user immediately leaves the femtocell, a problem known as temporary femtocell visitors. In this algorithm, the femtocell area and its surroundings are divided into sub-areas using positioning technology. Each mobile device transmits its movement history to the operating system (OS) of an analysis server periodically. Through movement pattern analysis based on pattern mining among these sub-areas, the next movement can be predicted. When a mobile device approaches the femtocell boundary, a check is executed to determine whether the next movement within the coverage of the femtocell in long enough. If so, it will perform the handoff.

The authors in [8] presented a handoff scheme based on the double threshold algorithm to reduce unnecessary handoffs between macrocells and femtocells. Their technique is based on a comparison of the signal levels of the source cell and the target cell with two thresholds, 1 and 2 . Threshold 1 is the minimum level required for the handoff from the macrocell to the femtocell, and Threshold 2 is the optimal level to improve the performance of the handoff. If the signal level of the source femtocell is lower than Threshold 1 and higher than Threshold 2, no handoff occurs. If it is not, then the handoff is executed. The algorithm also considers the duration of time that a mobile device maintains the signal above the required threshold.

\section{Motivational Paper}

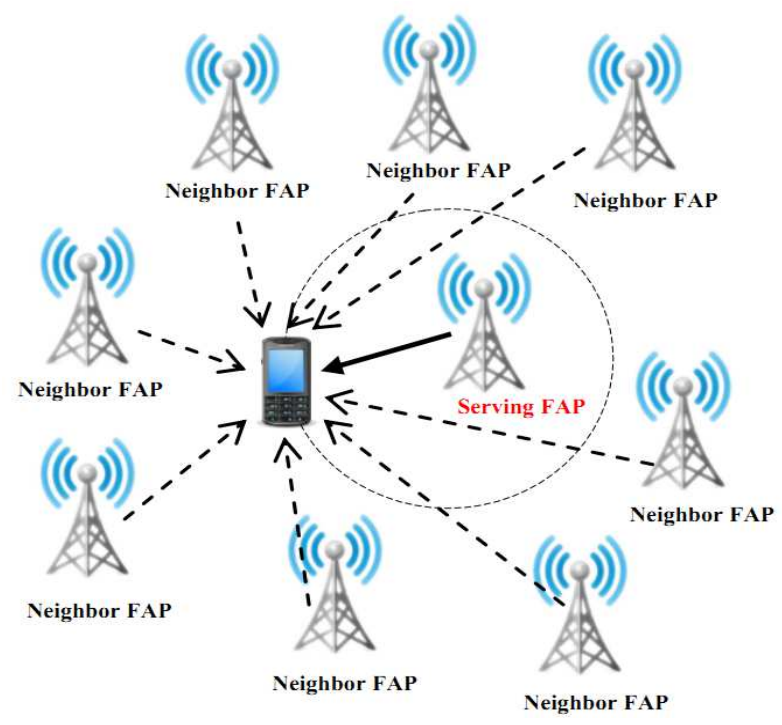

Fig. 3. Dense femtocellular network. 
Chowdhury et al. [6] proposed a schema to manage femtocell-to-femtocell handoffs in a dense femtocellular network, in which many femtocells are deployed within a small coverage area. Normally, in such a network, when the mobile station (MS) moves away from its serving FAP and the received signal from the serving FAP goes down, the MS receives many signals from the neighbor FAPs in order to establish the handoff. As a result of scanning the large number of FAPs, more power is consumed, and the overheads become more significant, affecting the performance of the entire network. Fig. 3 shows the deployment of a dense femtocellular network.

To overcome this issue, the authors in [6] proposed an algorithm that generates an appropriate list for the handoff with a minimum number of neighbor femtocells. Three main parameters are considered:

- The RSS from other FAPs.

- The frequencies detected from the serving FAP and the neighbor FAPs.

- Location information from the FAPs.

According to these parameters, femtocells are placed in two categories. The first category contains the FAPs from which the RSS is greater than or equal to a preset RSS threshold level. The FAPs that are in the second category are those from which the RSS is less than the threshold level or those serving FAPs and neighbor FAPs that use the same frequency. It is known that in dense femtocellular network deployment, two femtocells can't use same frequency unless they are far from each other. Therefore, any FAP that has the same frequency as the serving FAP will be eliminated from the neighbor list because it will be far from the serving FAP, and hence, the MS cannot establish a handoff with it. However, because there could be a hidden FAP within this category such that the MS cannot receive the signal due to some barrier between the MS and that FAP, location information is exchanged among the neighbor FAPs to detect and include the hidden FAPS on the list. The remaining FAPs are ignored.

The simulation results illustrated that the proposed scheme is able to minimize the number of neighbor FAP lists for the femtocell-to-femtocell handover. They also show that the probability for the hidden femtocell to not be on the neighbor femtocell list is reduced, while increasing the number of femtocells deployed. This is because the possibility that coordination is performed to obtain information about the hidden femtocell location increases. Thus, the handover failure rate decreases with an increasing number of femtocells.

\section{Citric}

Still, we observe that the generated neighbor FAPs list detailed in the previous section is not minimized enough to enhance the handoff between femtocells. In such a list, there could be many FAPs included, but those not located in the direction of the movement of the MS are useless. If the MS is moving north, the handoff will be established with one of the FAPs that are located in that direction; there is no need to consider any neighbor FAPs that are located in any other direction. Hence, this paper proposes a model to enhance the neighbor FAP list for femtocell-to-femtocell handoffs in a dense femtocell network based on the movement direction of the MS and the locations of the neighbor FAPs. The goal of this work is minimizing the list of neighbor FAPs for the handoff as much as possible, which will minimize handoff time and reduce the overhead of the entire network, ensuring better performance. As for choosing which FAP is best for the handoff, this will be left for future work.

\section{Proposed Model}

Usually, a femtocell-to-femtocell handoff is established with one of the FAPs located in an area in the same direction as the MS movement. To enhance the schema proposed by Chowdhury et al. [6], discussed earlier, we need to eliminate all FAPs that are not located in the direction of the MS movement from the neighbor 
FAP list. To do so, two parameters must be identified:

- The movement direction of the MS.

- The location of the neighbor FAPs in relation to the serving FAP.

As discussed earlier in relation to the mobility pattern prediction scheme presented by Byungjin et al. [7], the femtocell area and its surroundings are divided into sub-areas using positioning technology, which is being widely applied for location-based data services [9], [10] as shown in Fig. 4. This environment consists of a user mobility analysis server, femtocell access points, and mobile terminals. Each mobile device saves its consecutive movement history and transmits it to the user mobility analysis server periodically. The OS of this server will analyze and mine the mobile device's movement pattern and its velocity to predict its next consecutive movement using a generalized mobility pattern mining algorithm proposed by Yavas et al. [11]. From the mined mobility patterns, the server extracts some mobility rules that identify the next movement of the MS. The predicted movement is then delivered to the femtocell base station.

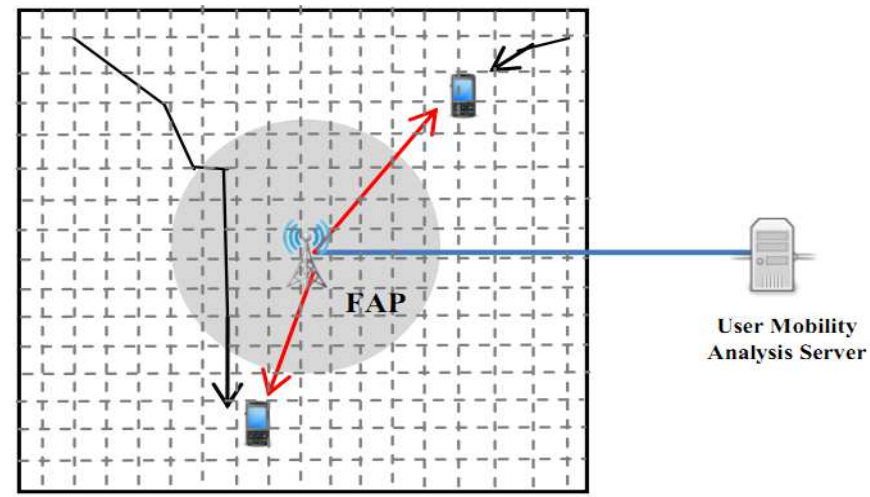

Fig. 4. MS movement prediction.

Fig. 5 shows the algorithm used by the operating system of the mobility analysis server to predict the movement direction of the mobile device. It consists of two sub-algorithms, Algorithm 1 for user mobility pattern mining and Algorithm 2 for generating mobility rules. In our proposed model, we will adapt this algorithm to predict the direction of the movement of the mobile station and use it as part of our total schema, in conjunction with the location of the neighbor femtocell access points, to enhance the femtocell list generated to establish the handoff in dense femtocell networks.

Here, we should note that there could an error in predicting the movement direction of the MS, especially if we take into consideration human behavior, which is not easy to predict. However, according to [6], the simulation shows that the error probabilities are always less than $6 \%$. This result is very reasonable, keeping in mind that there are no $100 \%$ perfect solutions. Dealing with a situation in which the movement prediction is incorrect is beyond the scope of this work.

To identify the other parameter, the location of the FAPs, we will use the OS of the same server, the user mobility analysis server. Here, a GPS system can be installed in the FAPs to enable the serving FAP to connect to a satellite and identify the location of each neighbor FAP included in the list generated from [6]. Note that the location of each FAP is fixed, so we will let the server OS read the list generated from [6]. According to the coordination of the neighbor FAPs, if the FAP is not located in the area of the direction of the MS's movement, it will be removed from the neighbor FAP list. Thus, minimizing the number of FAPs included in the list will reduce the need to scan a larger list and increase communication performance. In addition, identifying the locations of the FAPs means that the hidden FAPs are visible to the serving FAP. Thus, there is no need to perform the step illustrated earlier in [6] in which the location information is exchanged using the coordination of the neighbor FAPs to solve the problem of hidden FAPs. This will eliminate the overhead 
resulting from the communication between the serving FAP and the neighbor FAPs that was employed in [6] to detect the hidden FAPs.

\section{Algorithm 1 UserMobilityPatternMining $(H, S P \min , G)$}

$H$ : All the history of users in the database server

$S P \min :$ Minimum support value

$G$ : Surrounding sub-area graph

1: $S_{1} \leftarrow$ the candidate sub-area patterns which have a length of one

$2: k=1$

3: $U M P=\varphi$

4: $R=\varphi / /$ Initially the set is empty

5: while $S_{k}=\varphi$ do

6: $/ / S_{k}$ is the candidate length-k sub-area patterns

for all $s \in S_{k}$ do

for all history $h \in H$ do

if $s$ is a subsequence of $h$ then

s.count $=$ s.count + s.suppInc $/ /$ increment the support

.

11: end if

12: end for

end for

//choose the candidates which have enough support value

$L_{k}=\{s / s \in S k$, s.count $\geq S P \min \}$

$U M P=U M P \epsilon L k / /$ add these large patterns to set of UMP

$/ /$ Generate next length- $(\mathrm{k}+1)$ candidate patterns

for all $P \in L_{k}, P=<p 1, p 2, \ldots, p k>$ do

$V=\left\{v / v\right.$ is the neighbor of $\left.p_{k}\right\}$

for all $v$ do

//generate a candidate sub-area patterns

$S^{\prime}=\left\langle p_{1}, p_{2}, \ldots, p_{k}, v>\right.$

$S_{k+1}=S_{k+1} \in S^{\prime}$

end for

end for

$k=k+1$

27: end while

28: return $U M P$

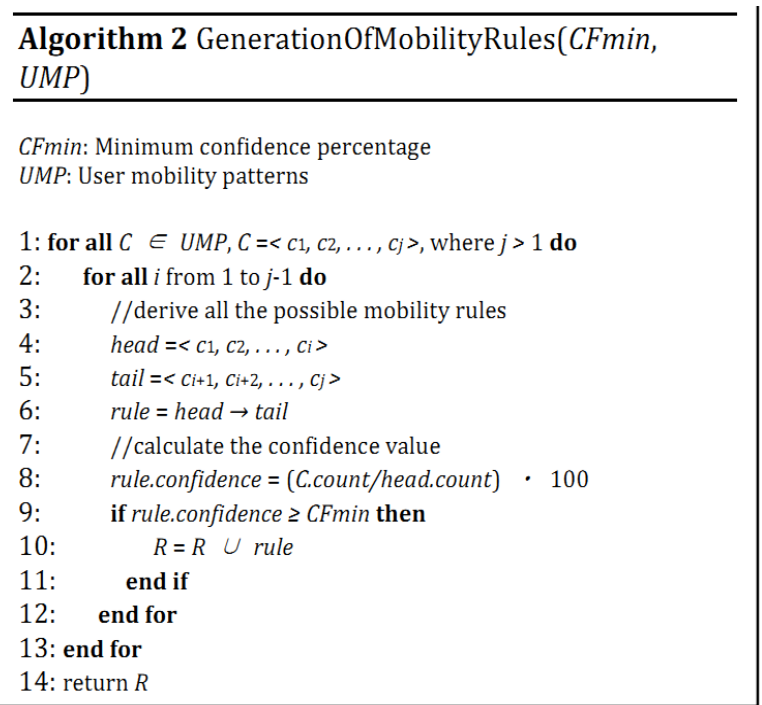

Fig. 5. Handover decision algorithm used to predict the movment direction of MS [7].

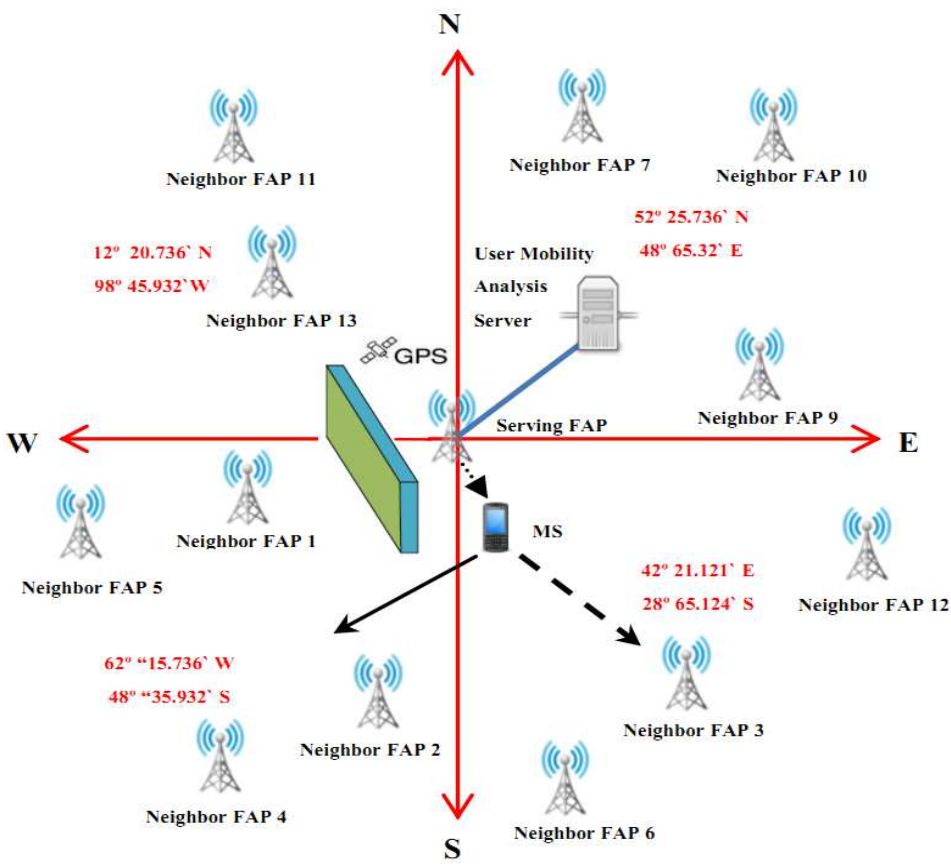

Fig. 6. Example of paper [6] enhancement. 
For an example of our enhancement, let us consider the femtocell network deployment shown in Fig. 6. The neighbor FAP list generated from [6] is [FAP1, FAP2, FAP3, FAP6, FAP7, FAP8] However, after applying our proposed model, assuming the movement of the MS in the direction of the arrow is predicted as shown (West-South), the neighbor FAPs list will shrink to [FAP1, FAP2], which is around 65\% less. If the direction of the movement is predicted as shown in the discreet arrow (East-South), the list will shrink to [FAP3], which is around $85 \%$ less. Note that, here, FAP1 is hidden from the serving FAP and can be made visible by using the user mobility analysis server and GPS, instead of applying the coordination technique used in [6].

\section{Conclusion}

One of the problems in dense femtocell networks is the handoff between femtocells. By using MS movement direction prediction and location information of neighbor FAPs, this paper achieved a minimized neighbor FAPs list for femtocell-to-femtocell handoff in a dense femtocell network.

\section{Future Work}

Instead of only minimizing the neighbor femtocell list used for the handoff, as proposed in our model, we can enhance the handoff by applying priority as an additional parameter to the list (e.g., according to the available bandwidth of each femtocell in the list). This will enable the MS to select the best option from the list for the handoff after the minimizing operation is performed.

\section{References}

[1] Internet World Statistics. From http://www.internetworldstats.com

[2] Femto Forum. About femtocells: What is femtocell? From http://www.femtoforum.org/femto/aboutFemtocells.php

[3] 3GPP. (April 2011). Security of home node B (HNB). Technical report, 3GPP TS 33.320.

[4] Wu, S. J. (2011). A new handover strategy between femtocell and macrocell for LTE-based network. Proceeding of Fourth International Conference on Ubi-Media Computing (pp. 203-208).

[5] Bai, T., Wang, Y., Liu, Y., \& Zhang, L. (2011). A policy-based handover mechanism between femtocell and macrocell for LTE-based networks. Proceedings of 2011 IEEE 13th International Conference on Communication Technology (pp. 916-920).

[6] Chowdhury, M. Z., Trung, B. M., \& Jang, Y. M. (2011). Neighbor cell list optimization for femtocell-to-femtocell handover in dense femtocellular networks. Proceedings of 2011 Third International Conference on Ubiquitous and Future Networks (pp. 241-245).

[7] Jeong, B., Shin, S., Jang, I., Sung, N. W., \& Yoon, H. (2011). Smart handover decision algorithm using location prediction for hierarchical macro/femtocell networks. Proceedings of 2011 IEEE Vehicular Technology Conference (pp. 1-5).

[8] Yang, G., Wang, X., \& Chen, X. (2011). Handover control for LTE femtocell networks. Proceedings of 2011 International Conference on Electronics, Communications and Control (pp. 2670-2673).

[9] Chintalapudi, K., Iyer, A. P., \& Padmanabhan, V. N. (Sept. 2010). Indoor localization without the pain. Proceeding of ACM Mobicom'10.

[10] Lee, M., Yang, H., Han, D., \& Yu, C. (April 2010). Crowd sourced radiomap for room-level place recognition in urban environment. Proceeding of IEEE PerCom Workshop on Smart Environments (SmartE 2010).

[11] Yavas, G., Katsaros, D., Ulusoy, O., \& Manolopoulos, Y. (Aug. 2005). A data mining approach for location prediction in mobile environments. Data \& Knowledge Engineering, 54, 121-146. 
Faisal A. Al-Shahin was born in Kuwait in 1979. He received his bachelor's degree in computer engineering, from the College of Engineering \& Petroleum, Kuwait University, Kuwait, 2002. Then he received the master's degree in computer engineering, from the College of Engineering \& Petroleum, Kuwait University, Kuwait, 2013.

In 2003, he joined the Legal Advice and Legislation Department, Council Of Ministers, Kuwait, as a beginner computer engineer, and in 2009, he became the head of the Technical Development Division of Information System Center. Since 2010, he has been with the Department of Information System Audit, State Audit Bureau of Kuwait, as a senior information system auditor. He is a co-author of "How to Diagram a Production Control System," presented in 2012 at the 12th International Conference on Control, Automation, and Systems (ICCAS 2012), October 17-21, Jeju Island, Korea. His current research interests include femtocell networks, wireless sensor networks, cloud computing, and information system security.

Eng. Al-Shahin is a life member the Kuwait Society of Engineering and a member of IEEE. 